\title{
Locating Accuracy of Shafts in V-blocks
}

\author{
V. E. Karpus ${ }^{a}$ and V. A. Ivanov ${ }^{b}$ \\ ${ }^{a}$ National Technical University “Kharkiv Polytechnic Institute”, Kharkiv, Ukraine \\ e-mail: karpus_vladislav@i.ua \\ ${ }^{b}$ Sumy State University, Sumy, Ukraine \\ e-mail: ivanov_vitalii@i.ua
}

\begin{abstract}
Analytical and experimental data are presented regarding the locating accuracy of stepped shafts in V-blocks, for drilling and milling machine tools. It is expedient to apply the proposed reusable V-block in modular reusable fixtures.
\end{abstract}

DOI: $10.3103 / \mathrm{S} 1068798 X 1202013 \mathrm{X}$

Modular reusable fixtures consist of reusable functional modules and combine the benefits of modular and reusable fixtures [1]. When using such fixture systems, the manufacturing costs of the individual components are reduced, while their flexibility and capabilities are increased. Effective use of such systems depends on maintenance of the required machining accuracy.

In the machining of shafts on drilling and milling machine tools, locating with respect to external cylindrical surfaces and the end is the most common approach. This relies on V-blocks of different structure, with angles $\alpha=60^{\circ}, 90^{\circ}$, and $120^{\circ}$. Most often, V-blocks with $\alpha=90^{\circ}$ are applied, since they afford greater stability than V-blocks with $\alpha=120^{\circ}$ and ensure a broader range of shaft diameters than those with $\alpha=60^{\circ}$ [2]. A set of standard rigid V-blocks (ten types) permits the locating of shafts with base surfaces of diameter 5-150 mm. Each type permits the locating of a small range $(10-15 \mathrm{~mm})$. To locate shafts of different size, the V-blocks are manually reconfigured, with considerable expenditure of setup time.

We propose the reusable V-block as a module for inclusion in modular reusable fixtures [1]. The locating elements take the form of disks with eccentric axes of rotation. A gear system permits simultaneous rotation of the supporting disks in different directions by a specified angle corresponding to the diameter of the shaft's cylindrical base surface.

The limiting diameters of the shaft's base surfaces depend on the radius $R$ of the supporting disks; the eccentricity $e$ (the amount by which the axis of rotation is displaced from the disk's center); and the distance $L$ between the axes of rotation of the supporting disks (Fig. 1). In consideration of the constraints on the machine tool's working zone, the radii of the supporting disks must be in the range $20-40 \mathrm{~mm}$. This permits the location of shafts with diameter $25-100 \mathrm{~mm}$. In these conditions, standard rigid V-blocks of five types may be replaced by the proposed reusable V-block.
The eccentricity $e$ must be between $R / 4$ and $R / 2$, while the maximum distance between the disks' axes of rotation is $L=3 R$. The position of the disks relative to the axes of rotation 1 is determined by the angle $\gamma$ between the axes of the shaft 2 and the supporting disk 3. It is inexpedient to select $\gamma>90^{\circ}$. In Fig. 1, we show the boundary positions of the supporting disks: $d_{\min }$ and $d_{\max }$ are the maximum and minimum diameters of the located shaft.

In analysis of the locating errors (Fig. 2), we consider the machining of flattened surfaces on a shaft in a horizontal milling machine tool. For dimension $h_{1}$, the locating error is

$$
\varepsilon_{\mathrm{b}_{h_{1}}}=O B-O_{1} B_{1}-O O_{1}=\frac{T d}{2}-O O_{1}
$$

where $O O_{1}$ is the maximum displacement of the shaft's axis as a result of the spread of the diameters among

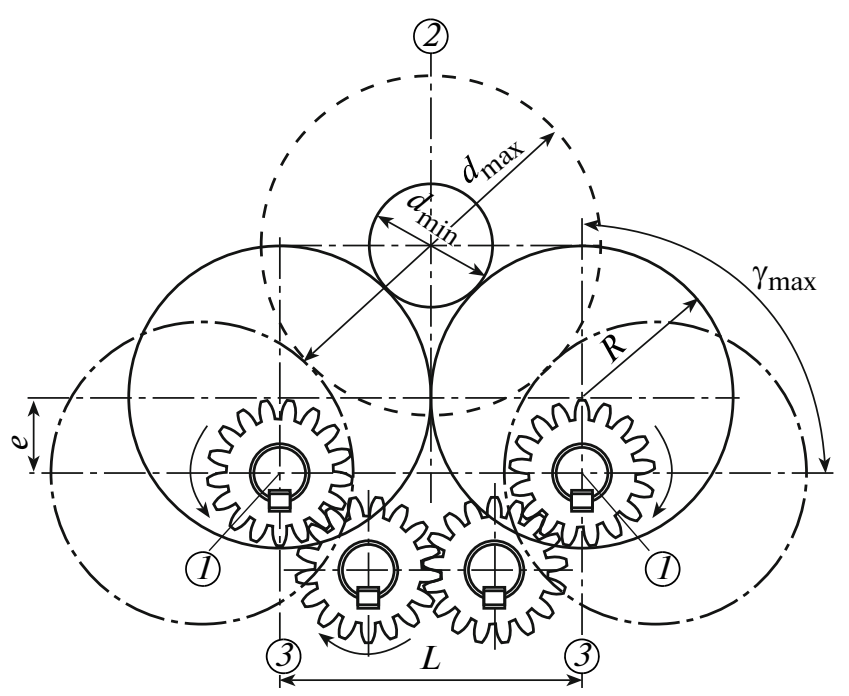

Fig. 1. Kinematic diagram of reusable V-block. 
the workpieces in the production batch, $\mathrm{mm}$; $T d$ is the tolerance on the diameter of the workpiece's base surface, $\mathrm{mm}$.

In turn

$$
\begin{gathered}
O O_{1}=O F-O_{1} F=\sqrt{O D^{2}-F D^{2}}-\sqrt{O_{1} D^{2}-F D^{2}} ; \\
O D=\frac{T d}{2}+\frac{d_{\min }}{2}+R ; \\
O_{1} D=O_{1} T_{1}+T_{1} D=\frac{d_{\min }}{2}+R ; \\
F D=F K+K D .
\end{gathered}
$$

Taking into account that $F K=L / 2$ and $K D=e \sin \gamma$, we may write

$$
\begin{aligned}
O O_{1} & =\sqrt{\left(\frac{T d}{2}+\frac{d_{\min }}{2}+R\right)^{2}-\left(\frac{L}{2}+e \sin \gamma\right)^{2}} \\
& -\sqrt{\left(\frac{d_{\min }}{2}+R\right)^{2}-\left(\frac{L}{2}+e \sin \gamma\right)^{2}} .
\end{aligned}
$$

Hence, the locating errors may be expressed in the following form:

for $h_{1}$

$$
\begin{aligned}
\varepsilon_{\mathrm{b}_{h_{1}}}= & \frac{T d}{2}-\left(\sqrt{\left(\frac{T d}{2}+\frac{d_{\min }}{2}+R\right)^{2}-\left(\frac{L}{2}+e \sin \gamma\right)^{2}}\right. \\
& \left.-\sqrt{\left(\frac{d_{\min }}{2}+R\right)^{2}-\left(\frac{L}{2}+e \sin \gamma\right)^{2}}\right) ;
\end{aligned}
$$

for $h_{2}$

$$
\begin{aligned}
\varepsilon_{\mathrm{b}_{h_{2}}}= & \frac{T d}{2}+\left(\sqrt{\left(\frac{T d}{2}+\frac{d_{\min }}{2}+R\right)^{2}-\left(\frac{L}{2}+e \sin \gamma\right)^{2}}\right. \\
& \left.-\sqrt{\left(\frac{d_{\min }}{2}+R\right)^{2}-\left(\frac{L}{2}+e \sin \gamma\right)^{2}}\right) ;
\end{aligned}
$$

for $h_{3}$

$$
\begin{aligned}
\varepsilon_{\mathrm{b}_{h_{3}}} & =\sqrt{\left(\frac{T d}{2}+\frac{d_{\min }}{2}+R\right)^{2}-\left(\frac{L}{2}+e \sin \gamma\right)^{2}} \\
& -\sqrt{\left(\frac{d_{\min }}{2}+R\right)^{2}-\left(\frac{L}{2}+e \sin \gamma\right)^{2}} .
\end{aligned}
$$

In Fig. 3, we show the locating error $\varepsilon_{\mathrm{b}_{h_{1}}}$ as a function of the accuracy of the workpiece's base surfaces and the angle $\gamma$ of the supporting disks. These graphs confirm that the influence of the base accuracy on the error of shaft locating in the reusable V-block is most significant and that this error increases somewhat with

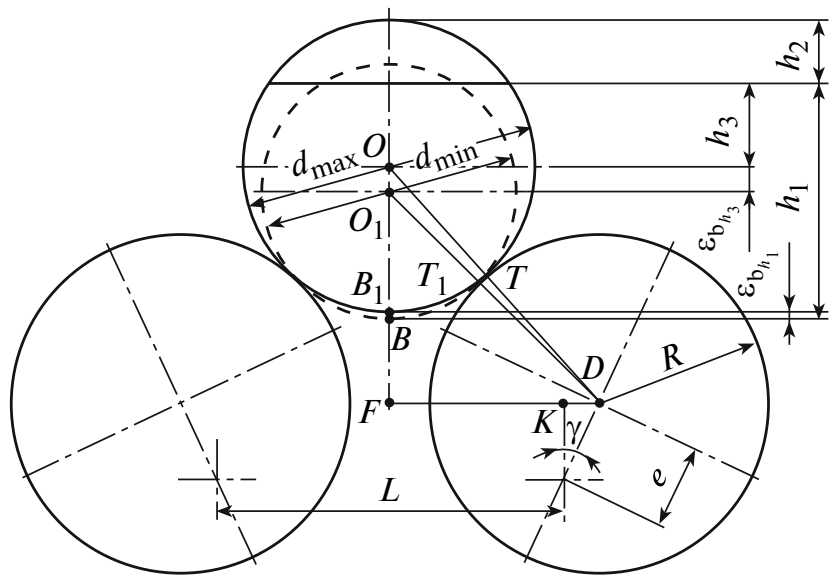

Fig. 2. Determining the locating error of a shaft in the reusable V-block.

increase in $\gamma$, especially in combination with low accuracy of the base surfaces.

Calculations show that, in the locating of shafts with diameter $30-100 \mathrm{~mm}$, when the base surfaces are machined with tolerances IT6-IT11, the locating error is $0.003-0.06 \mathrm{~mm}$ for $h_{1}, 0.019-0.266 \mathrm{~mm}$ for $h_{2}$, and $0.011-0.156 \mathrm{~mm}$ for $h_{3}$.

If we take account of the manufacturing accuracy of the fixture's components that adjust the V-block on switching to different workpieces, the basing errors of the shafts may be determined on the basis of dimensional analysis, by constructing a dimensional chain and performing minimax and probabilistic calculations. The formation of a dimensional chain for the milling of a flattered surface parallel to the plane of the machine tool's working table is illustrated in Table 1 (case 3).

The tolerances on the elements of the dimensional chain are assign with the working drawings of the prototype reusable V-block and with the tolerances on gear transmissions.

In minimax calculation, the tolerance on the master element is

$$
T A_{\Delta}=\sum_{i=1}^{10} T A_{i},
$$

where $T A_{i}$ is the tolerance for element $i$ in the dimensional chain.

In probabilistic calculation, the tolerance on the master element is

$$
T A_{\Delta}=\sqrt{\sum_{i=1}^{10}\left(K_{i}^{2} T A_{i}^{2}\right)},
$$

where $K_{i}$ is the relative scattering coefficient, which characterizes the deviation of the error distribution for 

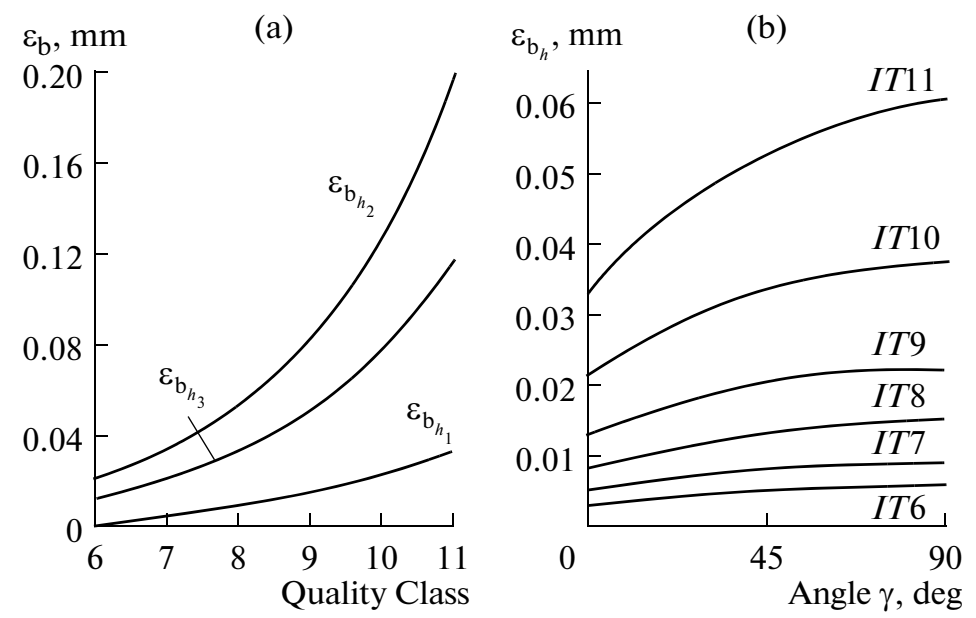

Fig. 3. Dependence of the locating error on the accuracy of the base surfaces (a) and the angle of the supporting disks (b).

element $i$ of the dimensional chain from normal distribution. (For a normal distribution, $K=1$.)

In the first case, we obtain $T A_{\Delta}=0.213 \mathrm{~mm}$; in the second, $T A_{\Delta}=0.07 \mathrm{~mm}$. For standard rigid V-blocks,
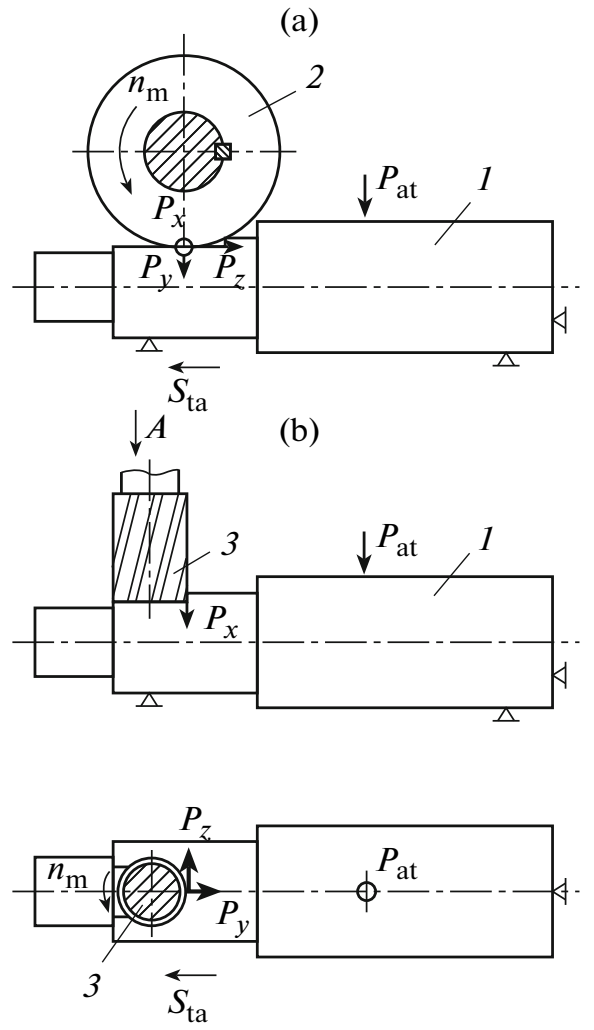

Fig. 4. Cutting forces with cylindrical (a) and end (b) mills: (1) stepped shaft; (2) cylindrical mill; (3) end mill; $P_{x}, P_{y}$, $P_{z}$, axial, radial, and tangential components of the cutting force, respectively; $P_{\mathrm{c}}$, clamping force; $n_{\mathrm{m}}$, mill speed; $S_{\mathrm{ta}}$, feed rate of machine-tool table. the corresponding values are 0.045 and $0.028 \mathrm{~mm}$ (case 1 in Table 1).

The tolerance $T A_{\Delta}$ on the master element when locating a shaft in V-blocks corresponding to mountable-and-dismountable fixtures is $0.119 \mathrm{~mm}$ for the minimax method and $0.056 \mathrm{~mm}$ for the probabilistic method (Table 1, case 5).

For an adjustable vise governed by two half-prisms (a gear-rack transmission), the tolerance on the master element is $0.162 \mathrm{~mm}$ for the minimax method and $0.074 \mathrm{~mm}$ for the probabilistic method (Table 1, case 7).

Table 1 also presents the calculated locating error for shafts in V-blocks of different design, with different positions of the milled surface. These results confirm that the locating error for shafts in the proposed reusable V-block is negligibly more than for other types of adjustable V-blocks. Thus, the proposed reusable V-block permits machining with acceptable accuracy, while the flexibility and adjustability are greater.

To determine the errors due to elastic deformation under the cutting forces, we conduct a finite-element analysis of the reusable and standard (rigid) V-blocks in the machining of flattened surfaces on a shaft by cylindrical (Fig. 4a) and end (Fig. 4b) mills.

We take account of the clamping forces and cutting forces corresponding to rough and semirough milling. The results of finite-element simulation show that, in semirough milling, the deformation of the workpieces is the same for both types of V-blocks: $0.002-0.008 \mathrm{~mm}$, depending on the position of the flattened surfaces (Table 2).

In rough milling, the elastic deformation is greater in the case of the cylindrical mill but is no more than $0.016 \mathrm{~mm}$ for the rigid V-block and $0.024 \mathrm{~mm}$ for the reusable V-block. The dimensional tolerance after rough milling is $T d=0.13 \mathrm{~mm}$ for workpieces with 
Table 1. Locating error of shafts in V-blocks

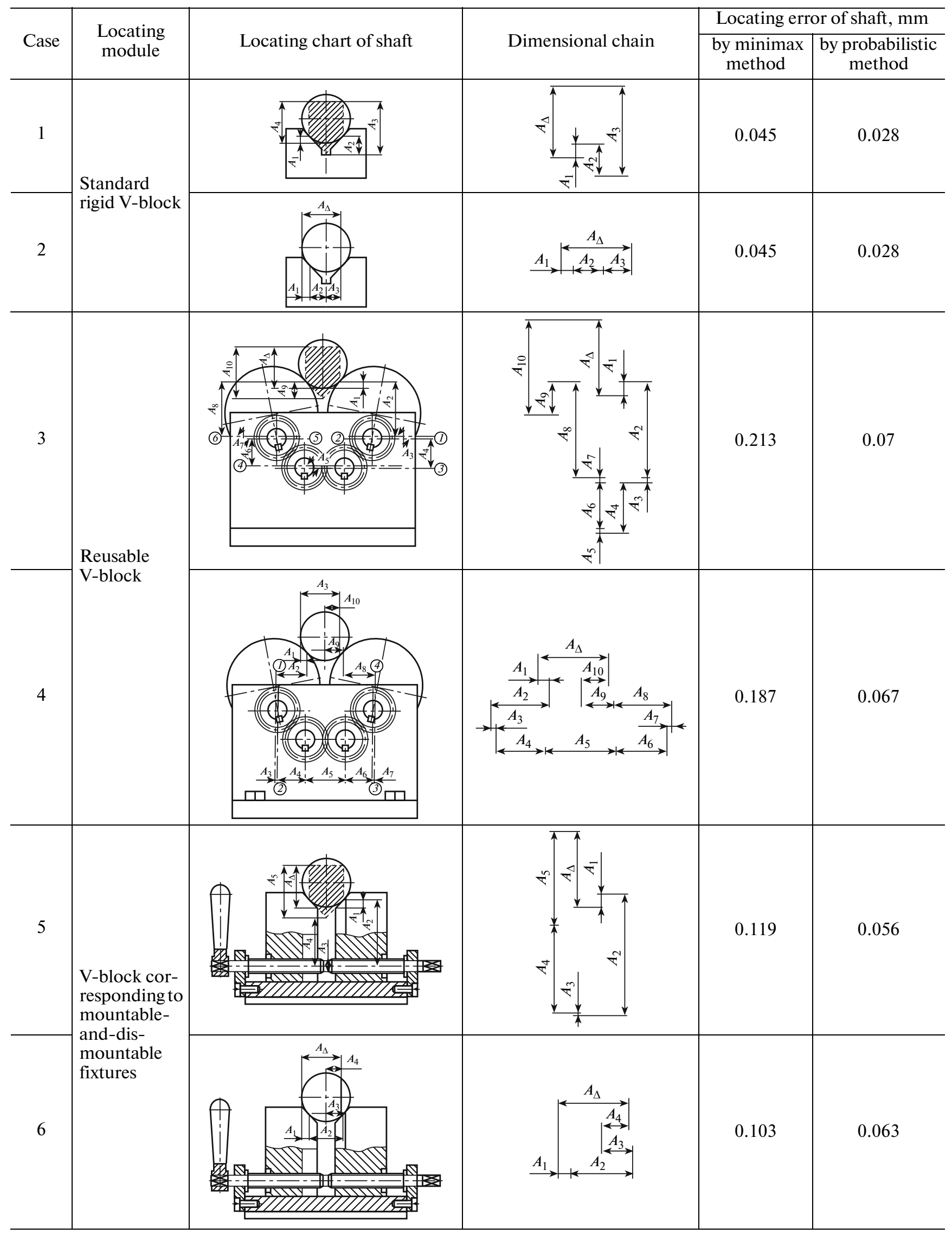


Table 1. (Contd.)

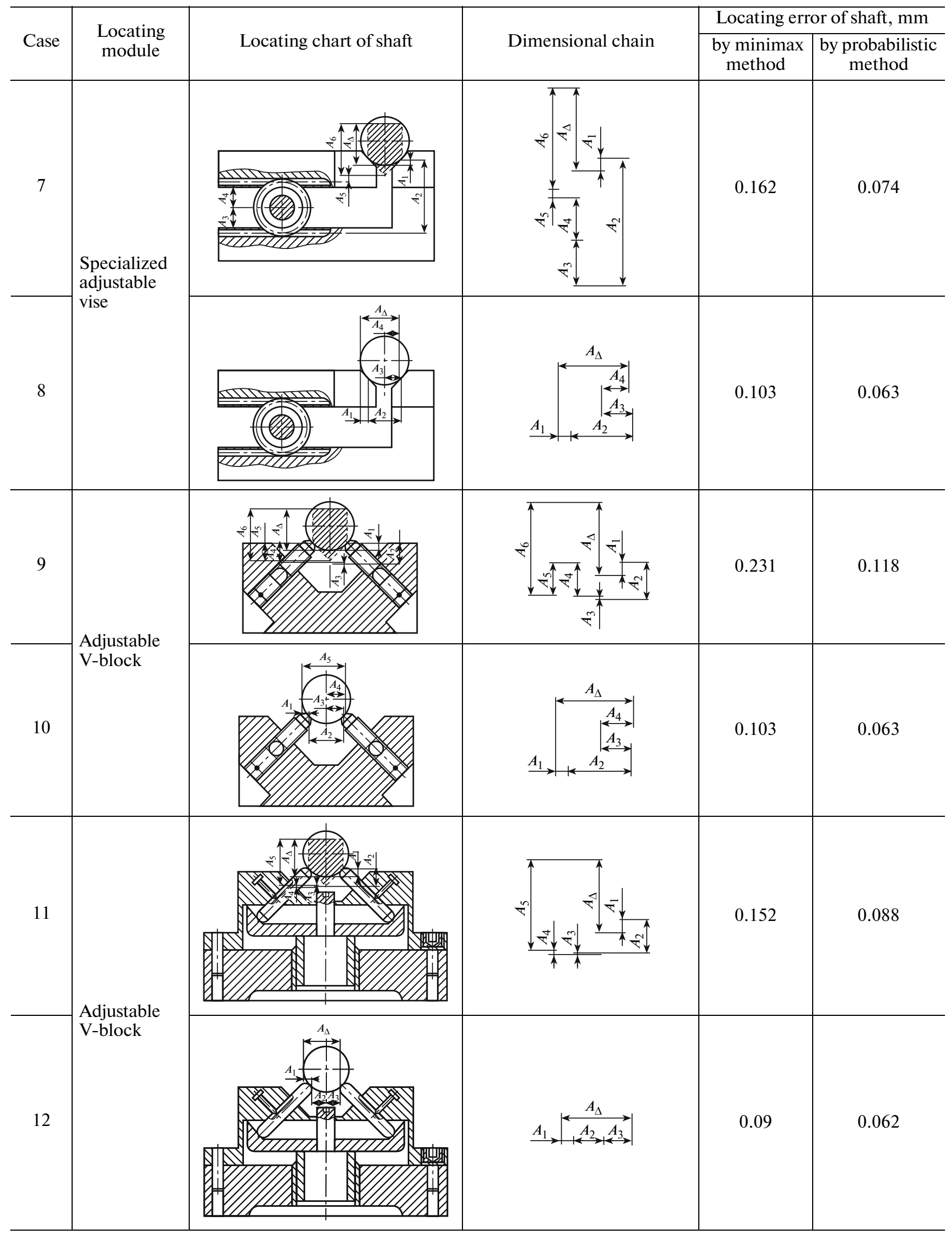


Table 2. Elastic displacements in milling flattened surfaces on shaft, $\mathrm{mm}$

\begin{tabular}{|c|c|c|c|c|c|c|}
\hline \multirow{4}{*}{ Locating module } & \multicolumn{6}{|c|}{ Machining point } \\
\hline & \multicolumn{2}{|c|}{ at cantilever } & \multicolumn{2}{|c|}{ above support } & \multicolumn{2}{|c|}{ between two supports } \\
\hline & \multicolumn{6}{|c|}{ at cutting depth $t, \mathrm{~mm}$} \\
\hline & 1 & 4 & 1 & 4 & 1 & 4 \\
\hline \multirow[t]{2}{*}{ Proposed reusable V-block } & \multicolumn{6}{|c|}{ When using a cylindrical mill } \\
\hline & 0.008 & 0.024 & 0.004 & 0.014 & 0.006 & 0.024 \\
\hline Standard rigid V-block & 0.008 & 0.016 & 0.004 & 0.008 & 0.006 & 0.015 \\
\hline \multirow[t]{2}{*}{ Proposed reusable V-block } & \multicolumn{6}{|c|}{ When using a face mill } \\
\hline & 0.004 & 0.008 & 0.002 & 0.005 & 0.004 & 0.008 \\
\hline Standard rigid V-block & 0.004 & 0.007 & 0.002 & 0.005 & 0.004 & 0.007 \\
\hline
\end{tabular}

diameter $18-30 \mathrm{~mm}, T d=0.16 \mathrm{~mm}$ for $30-50 \mathrm{~mm}$, and $T d=0.19 \mathrm{~mm}$ for $50-80 \mathrm{~mm}$.

Thus, the errors due to simulation of the deflected mode are considerably less than the tolerances for milling of the corresponding surfaces. Accordingly, the proposed reusable V-block fully complies with production conditions.

To verify our calculations, we conduct experiments on the machining accuracy of a stepped shaft in a prototype reusable V-block (Fig. 5). (a)

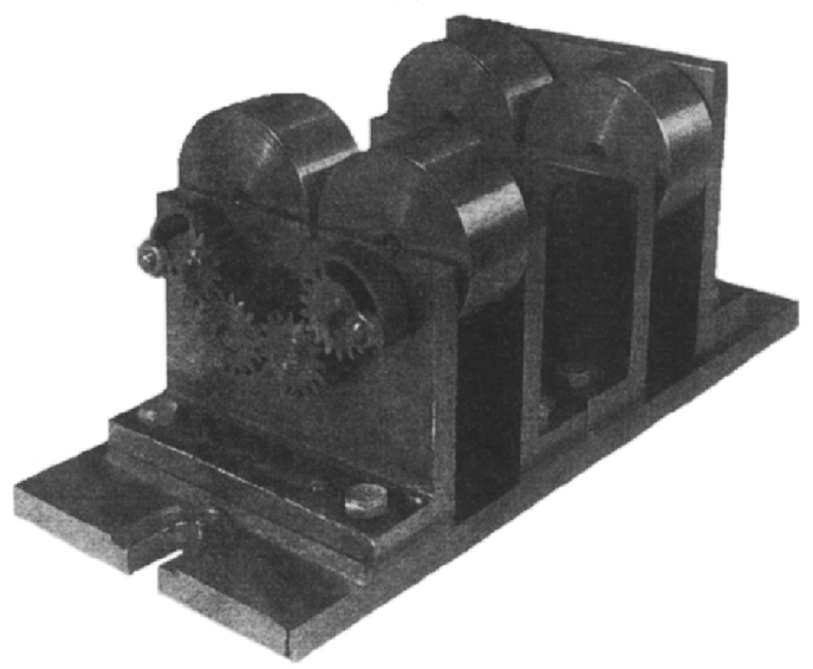

(b)

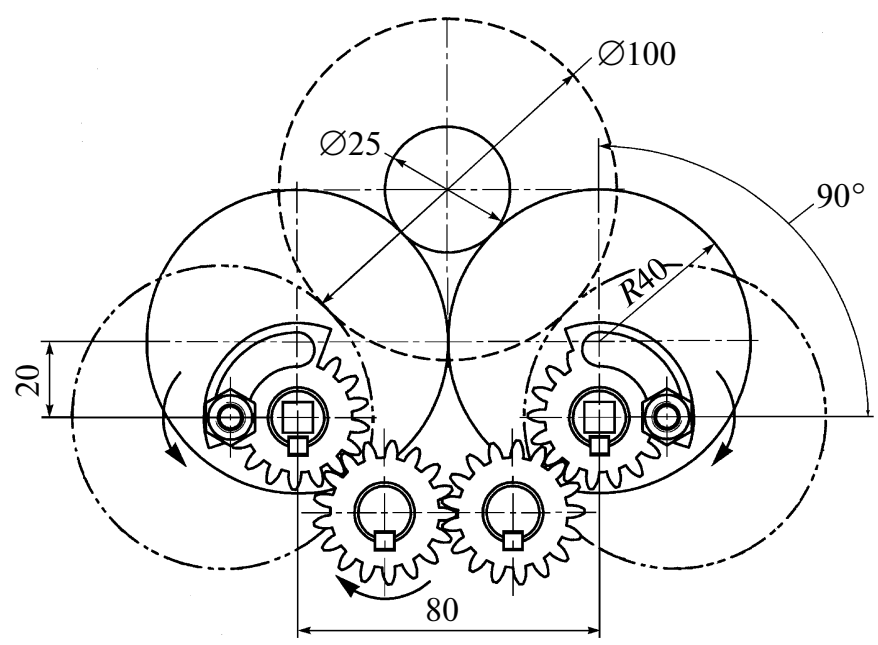

Fig. 5. Prototype reusable V-block (a) and mechanism regulating the locating elements (b). 
Milling operations corresponding to the analytical calculations are performed in horizontal and vertical milling machine tools.

In semirough milling with a cylindrical mill, the machining error is no more than $0.01 \mathrm{~mm}$. In rough milling, the error is greater, but no more than $0.02 \mathrm{~mm}$ for the rigid V-block and $0.03 \mathrm{~mm}$ for the reusable $\mathrm{V}$-block. When using an end mill, the error is no more than $0.01 \mathrm{~mm}$ in all cases.

Thus, the proposed adjustable reusable V-block permits considerable expansion of the capabilities and flexibility of machine tool fixtures, without significant loss of accuracy. Our research confirms the expediency in using such V-blocks in structure with modular reusable fixtures.

\section{REFERENCES}

1. Karpus, V.E. and Ivanov, V.A., Modular Reusable Fixtures, Vestn. Mashinostr., 2008, no. 11, pp. 46-50.

2. Korsakov, V.S., Osnovy konstruirovaniya prisposoblenii (Design Principles for Machine Tool Fixtures), Moscow: Mashinostroenie, 1983. 